\section{Prävention des ARDS mit Budesonid plus Formote- rol per inhalationem?}

Festic E et al. Randomized Clinical Trial of a Combination of an Inhaled Corticosteroid and Beta Agonist in Patients at Risk of Developing the Acute Respiratory Distress Syndrome. Crit Care Med 2017; 45: 798-805

Ein ARDS (Acute Respiratory Distress Syndrome) geht trotz Fortschritten in der supportiven Therapie immer noch mit einer hohen Komplikationsrate und Letalität einher. Es gibt bislang keine gesicherte pharmakologische Behandlung, die an der Ursache des ARDS angreift, aber möglicherweise könnte bei Hochrisikopatienten eine prophylaktische Therapie sinnvoll sein. Das haben nun Mediziner aus den USA in einer Pilotstudie untersucht.

Die Wissenschaftler um Emir Festic haben dazu zwischen September 2013 und Juni 2015 in 5 US-amerikanischen Zentren 61 Patienten mit hohem Risiko für die Entwicklung eines ARDS in eine randomisierte, Placebo-kontrollierte Doppelblindstudie aufgenommen.

Einschlusskriterien umfassten mindestens 1 ARDS-Risikofaktor, einen LIPSWert $\geq 4$ (LIPS: Lung Injury Prediction Study) und eine akute Hypoxämie, definiert als zusätzlicher Sauerstoffbedarf von mindestens $21 / \mathrm{min}$, um eine Sauerstoffsättigung zwischen $92 \%$ und $98 \%$ aufrechtzuerhalten. Patienten mit dem Vollbild eines ARDS wurden nicht in die Studie eingeschlossen.

Die Teilnehmer kamen direkt über die Notaufnahme und wurden innerhalb von $12 \mathrm{~h}$ nach Erstvorstellung nach dem Zufallsprinzip 1 von 2 Behandlungsgruppen zugewiesen:

- Inhalation von Budesonid (0,5 mg in $2 \mathrm{ml}$ ) und Formoterol ( $20 \mu \mathrm{g}$ in $2 \mathrm{ml})$ über einen handelsüblichen Vernebler 2-mal pro Tag über bis zu 5 Tage $(n=30)$ oder

- Inhalation 0,9\%iger Kochsalzlösung $(4 \mathrm{ml})$ als Placebo über den gleichen Zeitraum ( $n=31)$.
Diesem Ansatz liegt die Überlegung zugrunde, dass per inhalationem verabreichte $\beta$-Agonisten möglicherweise zu einer Stabilisierung der Pulmonalgefäße beitragen und die alveoläre FlüssigkeitsClearance verbessern können. Die Kortikosteroide wiederum sollen die inflammatorischen Veränderungen des ARDS abschwächen.

Als primären Endpunkt wählten die Wissenschaftler die Veränderung des Verhältnisses Sauerstoffsättigung zur inspiratorischen Sauerstoffkonzentration ( $\mathrm{S}$ / F) bis Tag 5; für diesen Wert wurden in der Vergangenheit gute Korrelationen mit dem Oxygenierungsindex gezeigt, auch wenn er noch nicht ausdrücklich validiert wurden. Sekundäre Endpunkte umfassten u.a. Entwicklung eines manifesten ARDS, Notwendigkeit einer Atemunterstützung (invasiv oder nicht invasiv), Dauer der Behandlung auf der Intensivstation und in der Klinik insgesamt.

Die Auswertung ergab zunächst, dass Patienten in der Interventionsgruppe älter waren (71 Jahre vs. 57 Jahre), geringere LIPS-Werte und seltener eine Schocksymptomatik aufwiesen (13\% vs. $47 \%$ ).

Die Zunahme des S/F-Verhältnisses

- war in der Interventionsgruppe bis Tag 5 signifikant höher,

- und die Trennung zwischen den beiden Behandlungsgruppen zeichnete sich ab Tag 2 ab.

Diese longitudinalen Veränderungen waren unabhängig von der Schocksymptomatik.

Insgesamt nahm das S/F-Verhältnis

- bei 18 Patienten der Interventionsgruppe $(62 \%)$ und

- bei 13 Patienten der Placebogruppe (43\%) um>20\% zu.

Bei weiterer Aufschlüsselung blieb dieser Unterschied aber nur bei Patienten ohne Schock (57\% vs. $44 \%$ ) und bei Patienten mit Pneumonie als Grunderkrankung (67\% vs. $44 \%$ ) signifikant.

Bei den sekundären Endpunkten fanden sich eine häufigere ARDS-Entwicklung bei Patienten der Placebogruppe (23\% vs. $0 \%$ ), eine häufigere maschinelle Beat- mung (53\% vs. $20 \%$ ) und ein längerer Intensivstations- und Krankenhausaufenthalt (im Median 6 Tage vs. 3 Tage und 6,5 Tage vs. 3,5 Tage). Alle diese Unterschiede verloren aber ihre Signifikanz, wenn im Hinblick auf das Vorliegen eines Schocks zu Studienbeginn adjustiert wurde.

Komplikationen durch die Studienbehandlung wurden nicht beschrieben.

\section{FAZIT}

Diese Ergebnisse zeigen bei ARDSHochrisikopatienten die Machbarkeit und Sicherheit eines prophylaktischen Behandlungsversuches mit Inhalation von Budesonid und Formoterol, fassen die Autoren zusammen. Die Daten dieser Pilotstudie müssen nun an größeren Kohorten bestätigt werden, und dabei müssen auch klinisch relevantere Endpunkte wie ARDS und Sterblichkeit als primäre Outcome-Parameter geprüft werden.

\section{Dr. Elke Ruchalla, Bad Dürrheim}

